\title{
The Association between Therapeutic Alliance and Individuals' Wish to Die or Live
}

\author{
Mariann Ring ${ }^{1 *}$, Sebastian Walther ${ }^{2}$, Anja C. Gysin-Maillart ${ }^{2,3}$ \\ ${ }^{1}$ Privatklinik Clienia Schlössli, Oetwil am See, Switzerland \\ ${ }^{2}$ Translational Research Centre, University Hospital of Psychiatry, University of Bern, Bern, Switzerland \\ ${ }^{3}$ Department of Medical Psychology and Medical Sociology, University of Leipzig, Leipzig, Germany \\ Email: *mariann.ring@clienia.ch
}

How to cite this paper: Ring, M., Walther, S., \& Gysin-Maillart, A. C. (2019). The Association between Therapeutic Alliance and Individuals' Wish to Die or Live. Psychology, 10, 1711-1725.

https://doi.org/10.4236/psych.2019.1012112

Received: July 3, 2019

Accepted: September 23, 2019

Published: September 26, 2019

Copyright $\odot 2019$ by author(s) and Scientific Research Publishing Inc. This work is licensed under the Creative Commons Attribution International License (CC BY 4.0).

http://creativecommons.org/licenses/by/4.0/

(c) (i) Open Access

\begin{abstract}
Objective: The relationship between therapeutic alliance and the individuals' expressed internal struggle about living or dying is investigated in this study. Additionally, the suicidal internal struggle and its relation to suicidal ideation are explored over time. Methods: Sixty suicide attempters from the ASSIP evaluation study were screened with the Helping Alliance Questionnaire and the Wish to Live and Wish to Die scales to assess therapeutic alliance and suicidal internal struggle at baseline, 6-, 12- and 24-months follow-up. Suicidal ideation was measured by the Beck Scale of Suicide Ideation. Results: Good therapeutic alliance and high satisfaction with therapy success are associated with a stronger wish to live in participants in the Attempted Suicide Short Intervention Program-ASSIP. Individuals' internal struggle was related to lower rates of suicidal ideation over time. Individuals with lower internal struggle (more wish to die) require much more attention to prevent the risk of suicidal crises. Limitations: Loss of information in our longitudinal investigation was due to low response rates at some follow-up time-points. Individuals who still have the wish to die after attempting suicide are the minority in our sample, indicating that these individuals are less likely to enter suicide prevention programs. Conclusion: The quality of therapeutic alliance is critical to reducing suicidal ideation.
\end{abstract}

\section{Keywords}

Internal Struggle, Therapeutic Alliance, Suicidal Ideation, ASSIP

\section{Introduction}

The prevalence of suicidal behaviour is a global public health problem. Over the past decades, research has made significant gains in better defining suicidal be- 
haviour, and numerous new suicide risk assessment instruments, approaches and models have been developed. However, despite these substantial advances in research and treatment of individuals experiencing suicidality, suicidal behaviour rates overall remain alarmingly high (World Health Organization, WHO, 2017).

Motives of suicidal behaviour are heterogeneous across affected individuals, as are suicidal thoughts (Jobes et al., 2004; Large et al., 2018). Reasons for living or dying have been investigated extensively in the prediction of suicidal ideation and suicidal behavior (Linehan, Goodstein, Nielsen, \& Chiles, 1983; Malone et al., 2000). Evidence showed that individuals with few reasons for living are at higher risk of suicidal ideation and attempting suicide (Galfalvy et al., 2006; Zhang, Law, \& Yip, 2011). Kovacs \& Beck (1977) explored the diversity in the suicidal mind and developed the "Internal Struggle Hypothesis" (ISH) that suggests that suicidal individuals do not hold a single unidirectional motivation but rather hold simultaneously both a wish to die and to live. They introduced the Wish-to-Die (WTD) and Wish-to-Live (WTL) self-report scales in order to test their hypothesis. Their study reported half of the patients to be embroiled in an internal debate; being ambivalent over whether to live or die. Moreover, patients who expressed both a wish to live and a wish to die showed lower intended lethality scores than patients who expressed solely a greater wish to die. To better understand the patient's wishes for dying might be used to correct and broaden the often constrained thinking of the suicidal individual. Alternativley, with ambivalent patients the therapist might put the focus on the patient's reasons for living and reinforcing them, and with patient who did not want to die at all, the focus might be on safe and new coping strategies. Brown et al. (2005a) expanded on the Internal Struggle Hypothesis and introduced the wish to die and wish to live difference score-the Internal Struggle (IS) score in order to find out whether there is a greater wish to die than wish to live constitutes as a unique risk factor for suicide. Analysis in their 10-year follow-up study with psychiatric outpatients showed that the Internal Struggle score rating is a distinct suicide risk factor. Furthermore, Harris et al. (2010) found strong support for the internal struggle hypothesis in their study. They asked both suicidal and nonsuicidal participants up front if they had ever engaged in an internal suicide debate. The vast majority indicated that they were at least once engaged in an internal life-death debate. In the sample of highly suicidal persons $94.5 \%$ confirmed to have such internal debates. O'Connor et al. (2012a) merged in their research the wish to die and wish to live ratings as done by Brown et al. (2005a); however, they included a third category, ambivalent about living or dying (AMB). They validated these three distinct groupings-wish to live (WTL), ambivalent about living or dying (AMB), and wish to die (WTD) in their analyses among high risk patient during psychiatric hospitalization. Analysis showed the importance of meaningful typologies related to suicidal ambivalence as they showed to be useful in identifying patient's needs for clinical treatments and interventions ( $\mathrm{O}^{\prime} \mathrm{Connor}$ et al., 2012b). 
People struggling with suicidal thoughts and behaviours often do not receive treatment (Downs \& Eisenberg, 2012). The main reasons are low perceived need and the wish to try solving the problems on their own (Bruffaerts et al., 2011). Metaanalysis demonstrated that psychosocial and behavioural interventions focussing directly on suicidal thoughts and behaviour are more effective in preventing suicide and suicide attemps (e.g. Brown et al., 2005b; Rudd et al., 2015) than interventions addressing the reduction of symptoms related to suicide behaviour (e.g. hopelessness, depression, anxiety) (Brodsky, Spruch-Feiner, \& Stanley, 2018; Meerwijk et al., 2016).

The Attempted Suicide Short Intervention Program (ASSIP), proven to be efficacious in reducing suicidal behavior (Gysin-Maillart, Schwab, Soravia, Megert, \& Michel, 2016b) and is to date one of the few psychotherapeutic treatments that addresses patients' suicidal thoughts and behavior directly. They were able to show in patients treated with the ASSIP that better therapeutic alliance correlated with lower suicidal ideation at 12-months follow-up (Gysin-Maillart, Soravia, Gemperli, \& Michel, 2016a). Moreover, the collaboration between the patient and therapist, particularly shared agreement on therapy goals, is important for the patient-rated satisfaction with the therapeutic relationship, the therapeutic success and the reduction of suicidal ideation over time (Ring, \& Gysin-Maillart, 2018/under review).

The present study attempts to explore the relationship between therapeutic alliance, and its two subscales: patients' satisfaction with therapeutic relationship and therapeutic success (Bassler, Potratz, \& Krauthauser, 1995) with patients' internal struggle over whether to die or live, following Brown et al. (2005a) and O'Connor et al. (2012a) methodology with suicide attempters' enroled in the ASSIP. In addition, the influence of patient's internal struggle on suicidal ideation is investigated over time. We expect that the better patients' quality of the therapeutic alliance and satisfaction with therapeutic relationship and therapy success is rated, the higher their internal struggle (IS) scores and thus the desire to live will be. Furthermore, we expect that the higher the individual internal struggle (IS) score is assessed, the lower suicidal ideation will be over time.

\section{Methods}

\subsection{Setting}

This study examined an archival dataset derived from a sample of 120 suicide attempters. The term attempted suicide used in the present study followed the definition as a "selfinflicted, potentially injurious behavior with a nonfatal outcome for which there is evidence (either explicit or implicit) of intent to die" (Silverman, Berman, Sanddal, O'Carroll, \& Joiner, 2007). They represent the treatment arm of a randomized controlled trial $(N=120)$ on the effectiveness of ASSIP in reducing suicidal behavior (ASSIP group: clinical assessment, treatment as usual and ASSIP; $N=60$; analyzed in this study) versus usual care (Control group: clinical assessment and treatment as usual; $N=60$ ) conducted 
by Gysin-Maillart et al. (2016b) at the University Hospital of Psychiatry Bern, which took place between June 2009 and December 2012. The study procedure was approved by the local ethics committee in Berne (registration number 144/08) in accordance with the Declaration of Helsinki (Rickham, 1964). Registration at ClinicalTrials.gov (NCT02505373) was done after completion of the study (All relevant Data Files of the ASSIP Evaluation Study, 2016 are openly available from the Dryad Digital Repository: http://dx.doi.org/10.5061/dryad.85nf3). The study sample was recruited from individuals who were admitted to the emergency unit of the Bern University Hospital following attempted suicide, who were seen by the duty psychiatrist at the University Hospital of Psychiatry Bern. Psychiatric hospital diagnoses were used based on the ICD-10 Classification of Mental and Behavioral Disorders (WHO, 2014). Individuals were excluded from the study when they had insufficient mastery of the German language, severe cognitive impairment, current psychotic disorder, antisocial personality disorder, and had their residence outside the hospital catchment area. When they fulfilled the inclusion criteria, written informed consent and agreement for video-recording the narrative interview was obtained and the three therapy sessions were conducted with the ASSIP group. Due to ethical and safety reasons participants in the control group were offered (Pearson, Stanley, King, \& Fisher, 2001), a single clinical interview, which included a suicide risk assessment using the SSF-II (Jobes, 2006). The present study will use data from the participants collected at the baseline session and at 6, 12, and 24 months follow-up. Further details have been published elsewhere (Gysin-Maillart et al., 2016b).

\subsection{Participants}

All participants included in the study had attempted suicide. The sixty participants in the ASSIP group, 24 (40\%) were male (mean age 39.42, SD = 15.25), and $36(60 \%)$ female (mean age $34.58, \mathrm{SD}=13.42)$; $55 \%$ of the individuals were diagnosed with major depression, $13 \%$ fulfilled the criteria for personality disorders, $28 \%$ for acute stress reaction, and $3 \%$ for substance abuse. Prior suicide attempts were reported by 26 (43\%) participants, of whom 10 were multiple repeaters. For further details on the demographic and clinical characteristics of the participants refer to Gysin-Maillart et al. (2016b).

\subsection{ASSIP Treatment Protocol}

The ASSIP is a brief therapy for working with individuals who have attempted suicide. The highly structured therapy consists of three sessions with an ongoing contact offer (Michel \& Gysin-Maillart, 2015). In the first Session a narrative interview is conducted in which patients are asked to tell their story of the suicide attempt in their own words (video-recorded narrative). In the second Session a video-playback is carried out, in order to identify relevant issues and triggers related to the suicidal crisis. In the third Session an individual case conceptualization and the personal safety strategies are revised collaboratively. These sessions 
are followed by regular semi-standardized letters over the course of two years.

\subsection{Therapists}

The brief therapy ASSIP, was provided by a psychiatrist and a clinical psychologist experienced in clinical suicide prevention and by two clinical psychologists with an average of 2 years in clinical practice, who were trained and certified in the ASSIP protocol.

\subsection{Measures}

The participants in this study completed a set of questionnaires after the baseline session and at 6-, 12- and 24 months. The Helping Alliance Questionnaire (Alexander \& Luborsky, 1986) measured therapeutic alliance ratings after the first—data used in this study - and the third session of the brief therapy ASSIP.

Helping Alliance Questionnaire-HAq-II (Alexander \& Luborsky, 1986; Bassler et al., 1995).

This is an 11-item self-rating questionnaire with 6-point Likert scales that range from $1=$ very inapplicable to $6=$ very applicable. It measures the strength of the patient-therapist therapeutic alliance and has a good internal consistency ( $\alpha=0.887$ ) (Alexander $\&$ Luborsky, 1986). In the present study the German version by Bassler et al. (1995) was used and their division of the therapeutic alliance (HAq) into two subscales: 1) "satisfaction with therapeutic relationship" (HAq_R) and 2) "satisfaction with therapeutic success" (HAq_S). The HAq_R scale rates the patient's experience of the relationship with the therapist-question: $1,6,7,8,9,10(\alpha=0.887)$ and the HAq_S scale measures the working alliance with the therapist based on the feeling of working together in a joint effort to the agreed therapy goals-question: $2,3,4,5,11(\alpha=0.840)$. Scale scores representing lower scores meaning less satisfaction with therapeutic relationship/therapy success and higher scores represent more satisfaction with therapeutic relationship/therapy success. Data used in the present study were collected at baseline session $(t 1)$.

Suicide Status Form-II-SSF-II (Jobes, 2006)

The SSF-II is a measure of suicidal ideation comprised of both quantitative and qualitative responses and is the primary assessment, treatment planning, and tracking tool used in the "Collaborative Assessment and Management of Suicidality" (CAMS) which was developed by Jobes (2006). The SSF-II has been psychometrically studied and found to be a valid (Cronbach ranging from 0.78 to 0.98 ) and reliable clinical assessment measure for suicide risk in previous studies (Conrad et al., 2009; Jobes, Jacoby, Cimbolic, \& Hustead, 1997). For our study purposes, we utilized both 8-point Likert scale responses of the participants' respective wish to live and their wish to die.

Wish to Live and Wish to Die scales-Internal struggle (IS) between wish to die and wish to live.

The SSF-II asks patients to answer the question: "I wish to live to the following extent" (WTL-score) and "I wish to die to the following extent" (WTD-score) us- 
ing a range of scores from $1=$ not at all to $8=$ very much. Both scales correspond with those originally employed by Kovacs \& Beck (1977) in their internal struggle hypothesis. Brown et al. (2005a) expanded the methodology by subtracting the Wish to Die scale score (WTD-score) from the Wish to Live scale score (WTL-score) for each patient, representing the Internal Struggle (IS) score of each individual: lower scores meaning $=$ more wish to die (weaker internal struggle) and higher scores meaning $=$ more wish to live (stronger internal struggle). In addition, following O'Connor et al. (2012a) the IS score (ranging from -7 to +7 in SSF-II) were trichotomized into the internal struggle groups: -7 to -3 wish to die (WTD-group), -2 to +2 ambivalent about dying or living (AMB-group) and +3 to +7 wish to live (WTL-group).

Beck Scale of Suicide Ideation-BSS (Beck \& Steer, 1991)

The BSS is a 21-items, self-report instrument used to measure the intensity of the patient's attitudes, behaviors and plans related to suicidal behavior during the past week, including the current day. All items consist of three response options, ranging from 0 to 2 . Five screening items reduce the length and the intrusiveness of the questionnaire for patients who are non-suicidal. Item 20 (prior suicide attempts) and 21 (severity of the suicide attempt) are not included in the score. The sum scores range from 0 to 38 points. The BSS is a well-standardized and widely used instrument among both researchers and clinicians and has demonstrated good criterion and convergent validity in previous research (Beck, Brown, \& Steer, 1997). In the present study the German version of the BSS is used (Wagner \& Maercker, 2008).

\subsection{Statistical Analysis}

In the present study we tested normality distribution with the Shapiro-Wilk test. The non-parametric method, the Spearman's rho test was used whenever appropriate. Bivariate correlations were performed to analyse the associations between the HAq-scale (HAq_R and HAq_S) at intial ASSIP session $(t 1)$ with the internal struggle (IS) at 6- , 12- and 24-months, which was calculated according the methodology described above. In addition, bivariate correlations were applied to test the association of internal struggle and the outcome measure suicidal ideation at baseline and across follow-up. Repeated measures ANOVA's tested for interactions, between groups and follow-up time-points. The Mauchly's test was applied and showed that the assumption of sphericity had been violated $\chi^{2}$ $(5)=21.489, p=0.001$, therefore degrees of freedom were corrected using Greenhouse-Geisser estimates of sphericity $(\varepsilon=0.553)$. All tests were two-tailed and a probability of false rejections $<0.05$ was considered statistically significant, applying a Bonferroni correction of multiple comparisons $\left(\alpha=0.05 / n_{\text {(tests) }}\right)$. Statistical analyses were carried out using the Statistical Package for Social Sciences (SPSS Statistics version 22).

\section{Results}

The dropout rate for the sixty adults and adolescent suicide attempters was 5\% 
$(N=3)$ at 12 months, and 7\% $(N=4)$ at 24 months, respectively. Reasons for withdrawal from the study was, e.g. refusal to continue the program due to change of ASSIP therapist during follow-up, and one recorded death by suicide occurring in an acute psychotic state. The number of missed assessments was $0 \%$ (baseline), 33\% (6 month), 42\% (12 month) and 12\% (24 month). At baseline session $(t 1) 60$ study participants reported an internal struggle (IS) score (mean score $2.65, \mathrm{SD}=0.58$ ). Subanalysis of the three subgroups (WTL, AMB, WTD) showed a frequency distribution of $42(70 \%)$ within the WTL group, $15(25 \%)$ in the AMB group, and $3(5 \%)$ within the WTD group. For the frequency distribution of the internal struggle ratings at 6-, 12-, and 24-months see Figure 1(a) ( $N$ ) and Figure 1(b) Percent (\%). Over time the distribution of cases between the three groups changed $\left(\chi^{2}(4)=21.67=p=0.000\right)$. At baseline, the majority of participants clearly wished to live, while ambivalence or wish to die was less common.

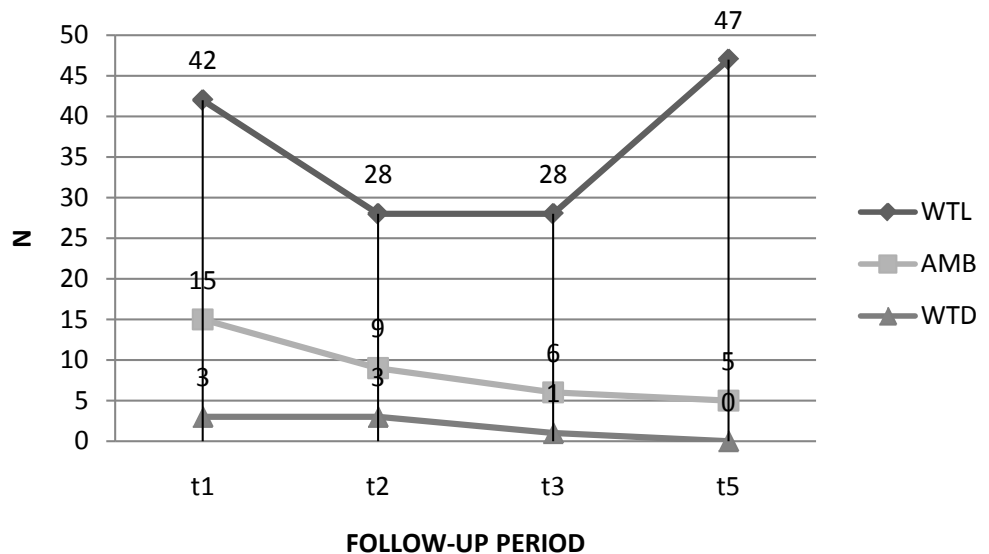

(a)

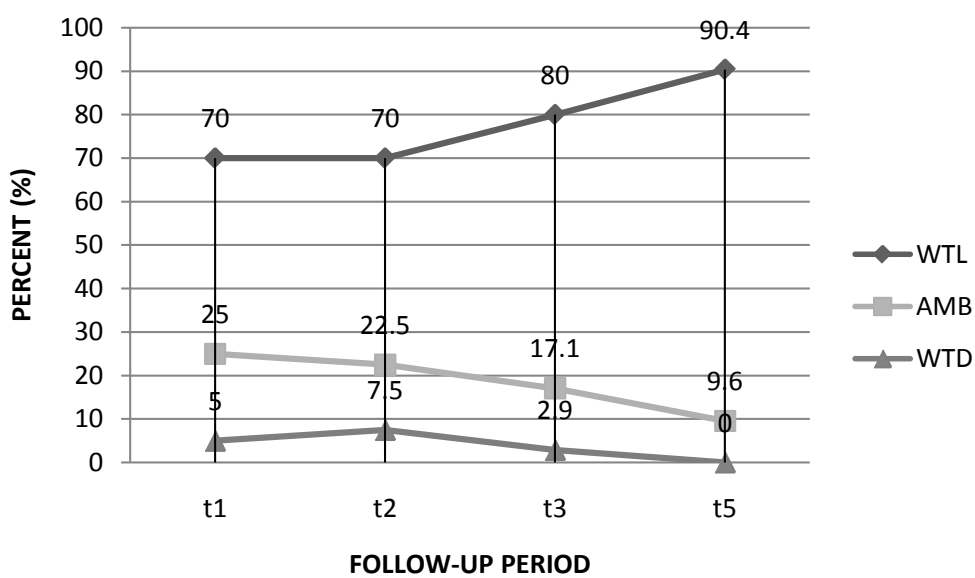

(b)

Figure 1. (a) Frequency Distribution $(N)$ of Internal Struggle to Die or Live Subgroups at Initial Session, $t 2$ (6 months), $t 3$ (12 months), and $t 5$ (24 months); WTL (Wish to Live), AMB (Ambivalent about Living or Dying), WTD (Wish to Die); $N: t 1=60 ; t 2=40 ; t 3=$ $35 ; t 5=52$; (b) Frequency Distribution (\%) of Internal Struggle to Die or Live Subgroups at Initial Session, $t 2$ (6 months), $t 3$ (12 months), and $t 5$ (24 months); WTL (Wish to Live), AMB (Ambivalent about Living or Dying), WTD (Wish to Die); $N: t 1=60 ; t 2=40 ; t 3=$ $35 ; t 5=52$. 
Over the follow-up period the number of participants in the WTL group declined at 6-, and 12-month, however, at 24-month the WTL again increased. The proportion of participants wishing to live steadily increased during follow-up. In comparison, both the AMB and WTD groups clearly remained smaller and declined over time.

Association between Therapeutic Alliance, its components: Patients' satisfaction with Therapeutic Relationship/Therapeutic Success and Internal Struggle during follow-up

We correlated the baseline HAq and its subscales HAq_R and HAq_S with the internal struggle score (IS) at four different time points (see Table 1). At initial ASSIP session $(t 1)$ we found substantial and statistically highly significant associations between HAq and the IS $(r=0.56, p=0.001)$, the HAq_R $(r=0.39 ; p=$ $0.002)$ and the HAq_S $(r=0.54 ; p=0.001)$. These results indicate that the better the therapeutic alliance, as well as patients' satisfaction with relationship and success were rated, the higher was the inner struggle (IS) score, indicating the wish to live rated by the participants. In turn, participants with the wish to die rated the quality of the therapeutic alliance to be lower. At 6 months $(t 2), \mathrm{HAq}$ showed a low but significant relation with the IS $(r=0.39, p=0.013)$, and significantly strong relations at 12 months $(t 3)(r=0.60, p=0.001)$ and at 24 months $(t 5)(r=0.37, p=0.007)$ follow-up. These show a good positive association between therapeutic allicance with the internal struggle ratings at 6 month, and strong associations at 12-, and 24-months. The IS, however, showed no relation with HAq_R at 6- and 24-month follow-up, and only at 12 month $(t 3)(r=$ $0.42, p=0.012)$ a weak one. Over all HAq_S scores were significantly associated with IS scores at 6 month $2(r=0.42, p=0.007)$, at 12 month $(r=0.61, p=0.001)$ and at 24 month $(r=0.42, p=0.002)$. These findings indicate that a good therapeutic alliance and a high satisfaction with therapeutic success at baseline are strongly related with higher internal struggle (IS) scores at follow-up showing a higher wish to live score.

\section{Relationship of Internal Struggle with Suicidal Ideation over time}

When comparing patient-ratings of the internal struggle (IS) score at baseline

Table 1. Bivariate correlations between Therapeutic Alliance (HAq), its two subgroups: Patients' Satisfaction with the Therapeutic Relationship (HAq_R)/Patients' Satisfaction with the Therapeutic Success (HAq_S) after the first Session and the Internal Struggle (IS) Score to Die or Live, at Initial Session $(t 1)$, at $6(t 2)$, at $12(t 3)$ and $24(t 5)$ months.

\begin{tabular}{ccccc}
\hline & ISscore $t 1$ & ISscore $t 2$ & ISscore $t 3$ & ISscore $t 5$ \\
\hline HAq & $r=0.56 ; p=0.001^{\star}$ & $r=0.39 ; p=0.013$ & $r=0.60 ; p=0.001^{\star}$ & $r=0.37 ; p=0.007$ \\
$\begin{array}{c}\text { HAq_ } \\
\text { Relationship }\end{array}$ & $r=0.39 ; p=0.002^{\star}$ & $r=0.22 ; p=0.179$ & $r=0.42 ; p=0.012$ & $r=0.24 ; p=0.086$ \\
HAq_Success & $r=0.54 ; p=0.001^{*}$ & $r=0.42 ; p=0.007$ & $r=0.61 ; p=0.001^{\star}$ & $r=0.42 ; p=0.002^{*}$ \\
\hline
\end{tabular}

* = Significant with Bonferroni adjustment. Note: Abbreviations: HAq, Therapeutic Alliance Questionnaire (Alexander \& Luborsky, 1986), after the first session, 1 = very inapplicable to 6 = very applicable; HAq_R: Therapeutic relationship scale, after the first session; HAq_S: Therapeutic success scale, after the first session; IS score: Internal struggle to die or live, part of the Suicide Status Form-II-SSF-II (Jobes, 2006), high scores $=$ Wish to live, low scores $=$ Wish to die; $N=t 1=60 ; t 2=40 ; t 3=35 ; t 5=52$. 
( $t 1$ ) with suicidal ideation at initial session ( $t 1$ ) (BSS $t 1$ ), 6- (BSS $t 2$ ), 12- (BSS t3), and 24- (BSS t5) months (Table 2), we found highly significant negative correlations between the internal struggle and suicidal ideation over all measureing points, at baseline $(t 1)(r=-0.62, p=0.001)$, at $6-(r=-0.54, p=0.001)$, and at 12 -months $(r=-0.55 ; p=0.001)$, and at 24-months $(r=-0.38 ; p=$ $0.005)$. This finding suggests a highly significant association between individuals internal struggle and suicidal ideation over 24 months, in other words, higher interal struggle scores, meaning a higher wish to live, were stongly associated with lower scores in suicidal ideation ratings over time and lower internal struggle scores meaning a higher wish to die, were associated with more suicidal ideation in the course of time.

Repeated measures ANOVA found a significant reduction in suicidal ideation over time $\left.\left(\mathrm{F}_{1.659,24.888}\right)=1.057, p=0.001\right)$, but no group effect between suicidal ideation and internal struggle $\left.\left(\mathrm{F}_{13.274,24.888}\right)=0.175, p=0.119\right)$. Follow up comparisons indicated that there were pairwise significant differences between baseline and 12- and 24-months, $p<0.05$, and between 6- and 24-months, $p=0.002$.

\section{Discussion}

The aim of the present study was to investigate the association between therapeutic alliance, its two components "satisfaction with therapeutic relationship/satisfaction with therapy success" and the internal struggle between wish to die and wish to live. Further, we explored the relation between the individuals' internal struggle and suicidal ideation over time. To address this question, the treatment arm of the ASSIP evaluation study (Gysin-Maillart et al., 2016b) was used.

Our findings demonstrate that the Wish to Live (WTL) group was holding by far most of the participants at initial session, whereas the ambivalent about dying or living (AMB) group and the Wish to Die (WTD) were smaller. This finding is not reflected in O'Connor et al. (2012a) study where the three groups were quite evenly distributed at baseline. This might not be a contradiction and can probably be explained due to the different study samples. In our study the participants were suicide attempters, who underwent a suicide-specific intervention, whilst O'Connor et al. (2012a) study included individuals who expressed current suicidal ideation in the previous 48 hours. In suicidal crisis the focus on long-term

Table 2. Bivariate correlations between the Internal Struggle (IS) Score to Die or Live, at Initial Session $(t 1)$, after the first Session and Suicidal Ideation at initial Session $(t 1)$, at 6 $(t 2)$, at $12(t 3)$ and $24(t 5)$ months.

\begin{tabular}{|c|c|c|c|c|}
\hline & BSS $t 1$ & BSS $t 2$ & BSS $t 3$ & BSS $t 5$ \\
\hline ISscore & $r=-0.62 ; p=0.001^{\star}$ & $r=-0.54 ; p=0.001^{\star}$ & $r=-0.55 ; p=0.001^{\star}$ & $r=-0.38 ; p=0.005$ \\
\hline
\end{tabular}

* = Significant with Bonferroni adjustment. Note. ISscore: Internal struggle score to die or live, part of the Suicide Status Form-II-SSF-II (Jobes, 2006), high scores = Wish to live, low scores = Wish to die; BSS: Beck Scale Ideation $($ Beck \& Steer, 1991), 0 point $=$ not suicidal to 38 points $=$ highly suicidal; $N=t 1=59 ; t 2=$ $44 ; t 3=36 ; t 5=54$ 
life goals is lost (Rudd, Joiner, \& Rajab, 2001) hence a temporarly increased wish to die emerges, whereas, after a suicide attempt the unbearable inner pain and tension decreases and life-oriented goals may soon reemerge (Michel \& Valach, 1997). In addition, by addressing the processes and patterns, involved in the development of the patient's suicidal crisis, as done in ASSIP, life-oriented goals become again a greater focus.

The quality of the therapeutic alliance and superior patient satisfaction with therapeutic success at baseline showed to be strongly related with higher internal struggle (IS) scores showing a stronger wish to live across all four measuring points. Thus, participants who are satisfied with the therapeutic relationship will be more likely to have stronger wish to live. A possible interpretation of these results suggests that during the course of ASSIP, participants learn in a secure alliance with the therapist to tell their own story how it came about to attempt suicide and due to the acquirement of tools and strategies for any future suicidal crisis they experience an improved sense of control and their expectation of empowerment are met (Constantino, Arnow, Blasey, \& Agras, 2005; Ring \& Gysin-Maillart, 2018/under review) thus participants are no longer passive in the sense of learned helplessness response patterns as described by Seligman (1975) or experience suicide as the only option to escape mental pain, which appears to be unbearable (Shneidman, 1993). In addition, this seems to be consistent with findings from research, which suggests that therapeutic alliance ratings in the third therapy session are a good predictor of adherence to treatment and outcome (Horvath \& Symonds, 1991; Saltzman, Luetgert, Roth, Creaser, \& Howard, 1976). Although this study was using data only after the first ASSIP session, the patient-centered collaborative approach in ASSIP seems to sustain participants' stronger wish to live over time. Whether the wish to die at inital session could have been transformed into a more live-oriented wish through the brief therapy ASSIP would have to be subject to further analysis.

Contrary to previous literature on therapeutic relationship across different kind of psychiatric settings and treatments (Castonguay \& Beutler, 2006; Martin, Garsle, \& Davis, 2000; McCabe \& Priebe, 2004; Priebe, Richardson, Cooney, Adedeji, \& McCabe, 2011) and on the important role of relationship in suicidal states (Etzersdorfer, 2012; Jobes, 1995, 2000; Joiner et al., 2002), we found that greater patients' satisfaction with the therapeutic relationship was only significantly associated with higher internal struggle (IS) scores at intial session, and weakly one at 12 months. These findings suggest that the therapist acting in his professional role is associated with the patients' satisfaction with the therapeutic relationship only at initial session serving for better engagement and adherence in therapy. However, over time the focus of importance seems to be remaining more on change of symptoms, behavior and satisfaction with therapy success.

Remarkably, participants' who showed high internal struggle (IS) scores correlated significantly high with lower suicidal ideation (BSS) at all four measuring points. Indicating that the higher the internal struggle meaning a stronger wish to live was rated, the lower suicidal ideation appeared. Our findings are sup- 
ported by prior research in which highly suicidal individuals were engaging more often and more severely in an internal suicidal debate, hence a struggle than non-suicidal or less suicidal persons (Harris, McLean, Sheffield, \& Jobes, 2010) and was also shown in Kovacs \& Beck (1977) study that when the subjectively perceived wish to die is much greater than the wish to live severity of suicidal intent becomes stronger. High internal struggle scores seem to be associated with a wish to continue living and a reduced suicidal ideation. These correspond with our results, which showed that high internal struggle scores were releated to lower rates in suicidal ideation not only at initial session, but also over time. This might be due to normal reduction of suicidal ideation over time.

There are several limitations to note in this study. A well known problem with treatment studies with long-term follow-up is missing data due to low response rates. In the present study the return of the questionnares was at certain time-points (e.g. 12 month $(t 3), N=35$ ) limited, and therefore reducing the study power of the quantitave approach and the gain of information of the longitudinal investigation. However, it was nevertheless possible to conduct the targeted explorations and that our response rates were in accordance to others known in research (Yang \& Lester, 2006). The participants analysed were weighted towards high internal struggle scores (stronger wish to live), whereas the patients with lower internal struggle (more with to die) might have refused more often to take part in the present study. The relatively small $(N=60)$ sample size and the unevenly distribution of participants across the three groupings limited the statistical power, which is particularly problematic for the repeated measures ANOVA on suicidal ideation over time. Further limitations of the ASSIP study have been published elsewhere (Gysin-Maillart et al., 2016b; Ring \& Gysin-Maillart, 2018/under review).

\section{Conclusion}

In summary, results from individuals after attempting suicide who participated in the ASSIP - a manual-based brief therapy-indicated that a good therapeutic alliance and in particular a high satisfaction with therapeutic success at baseline are strongly related with higher internal struggle (IS) scores showing a stronger wish to live. In addition, the individuals' internal struggle is significantly associated with lower rates of suicidal ideation over time. This study indicates, that individuals with low internal struggle, showing a strong wish to die, need special attention and interventions tailored to them, in order to prevent the risk of suicidal crises in the future.

\section{Funding}

There were no sources of funding for this research.

\section{Conflicts of Interest}

The authors declare no potential conflicts of interest with respect to the research, 
authorship, and/or publication of this article.

\section{References}

Alexander, L. B., \& Luborsky, L. (1986). The Penn Helping Alliance Scales. In L. S. Greenberg, \& W. M. Pinsoff (Eds.), In the Psychotherapeutic Process: A Research Handbook (pp. 325-366). New York: Guilford Press. https://doi.org/10.1037/t02248-000

Bassler, M., Potratz, B., \& Krauthauser, H. (1995). Der Helping Alliance Questionnaire (HAQ) von Luborsky. Möglichkeiten zur Evaluation des therapeutischen Prozesses von stationärer Psychotherapie. Psychotherapeut, 40, 23-32.

Beck, A. T., \& Steer, R. A. (1991). Manual for the Beck Scale for Suicide Ideation. San Antonio, TX: Psychological Corporation.

Beck, A. T., Brown, G. K., \& Steer, R. A. (1997). Psychometric Characteristics of the Scale for Suicide Ideation with Psychiatric Outpatients. Behaviour Research and Therapy, 35, 1039-1046. https://doi.org/10.1016/S0005-7967(97)00073-9

Brodsky, B. S., Spruch-Feiner, A., \& Stanley, B. (2018). The Zero Suicide Model: Applying Evidence-Based Suicide Prevention Practices to Clinical Care. Front Psychiatry, 9, 33. https://doi.org/10.3389/fpsyt.2018.00033

Brown, G. K., Steer, R. A., Henriques, G. R., \& Beck, A. T. (2005a). The Internal Struggle between the Wish to Die and the Wish to Live: A Risk Factor for Suicide. American Journal of Psychiatry, 162, 1977-1979. https://doi.org/10.1176/appi.ajp.162.10.1977

Brown, G. K., Ten Have, T., Henriques, G. R., Xie, S. X., Hollander, J. E., \& Beck, A.T. (2005b). Cognitive Therapy for the Prevention of Suicide Attempts: A Randomized Controlled Trial. JAMA, 294, 563-570. https://doi.org/10.1001/jama.294.5.563

Bruffaerts, R., Demyttenaere, K., Hwang, I., Chiu, W. T., Sampson, N., Kessler, R. C., Nock, M. K. et al. (2011). Treatment of Suicidal People around the World. The British Journal of Psychiatry, 199, 64-70. https://doi.org/10.1192/bjp.bp.110.084129

Castonguay, L. G., \& Beutler, L. E. (2006). Principles of Therapeutic Change That Work. New York: Oxford University Press. https://doi.org/10.1093/med:psych/9780195156843.001.0001

Conrad, A. K., Jacoby, A. M., Jobes, D. A., Lineberry, T. W., Shea, C. E., Ewing, T., \& Kung, S. (2009). A Psychometric Investigation of the Suicide Status Form II with a Psychiatric Inpatient Sample. Suicide and Life-Threatening Behavior, 39, 307-320. https://doi.org/10.1521/suli.2009.39.3.307

Constantino, M. J., Arnow, B. A., Blasey, C., \& Agras W. S. (2005). The Association between Patient Characteristics and the Therapeutic Alliance in Cognitive-Behavioral and Interpersonal Therapy for Bulimia Nervosa. Journal of Consulting and Clinical Psychology, 73, 203-211. https://doi.org/10.1037/0022-006X.73.2.203

Data File of the ASSIP Evaluation Study (2016). Dryad Digital Repository.

Downs, M. F., \& Eisenberg, D. (2012). Help Seeking and Treatment Use among Suicidal College Students. Journal of American College Health, 60, 104-114. https://doi.org/10.1080/07448481.2011.619611

Etzersdorfer, E. (2012). Der therapeutische Umgang mit Suizidalität: Was kann die Psychoanalyse beitragen. Psychotherapie im Dialog-Suizid, 2, 31-33. https://doi.org/10.1055/s-0032-1304974

Galfalvy, H., Oquendo, M. A., Carballo, J. J., Sher, L., Grunebaum, M. F., Burke, A., \& Mann, J. J. (2006). Clinical Predictors of Suicidal Acts after Major Depression in Bipolar Disorder: A Prospective Study. Bipolar Disorders, 8, 586-595. 
https://doi.org/10.1111/j.1399-5618.2006.00340.x

Gysin-Maillart, A. C., Soravia, L. M., Gemperli, A., \& Michel, K. (2016a). Suicide Ideation Is Related to Therapeutic Alliance in a Brief Therapy for Attempted Suicide. Archives of Suicide Research, 21, 113-126. https://doi.org/10.1080/13811118.2016.1162242

Gysin-Maillart, A., Schwab, S., Soravia, L., Megert, M., \& Michel, K. (2016b). A Novel Brief Therapy for Patients Who Attempt Suicide: A 24-Months Follow-Up Randomized Controlled Study of the Attempted Suicide Short Intervention Program (ASSIP). PLOS Medicine, 13, e1001968. https://doi.org/10.1371/journal.pmed.1001968

Harris, K. M., McLean, J. P., Sheffield, J., \& Jobes, D. (2010). The Internal Suicide Debate Hypothesis: Exploring the Life versus Death Struggle. Suicide and Life-Threatening Behavior, 40, 181-192. https://doi.org/10.1521/suli.2010.40.2.181

Horvath, A. O., \& Symonds, B. D. (1991). Relation between Working Alliance and Outcome in Psychotherapy: A Meta-Analysis. Journal of Counseling Psychology, 38, 139-149. https://doi.org/10.1037/0022-0167.38.2.139

Jobes, D. A. (1995). The Challenge and the Promise of Clinical Suicidology. Suicide and Life-Threatening Behavior, 25, 437-449.

Jobes, D. A. (2000). Collaborating to Prevent Suicide: A Clinical-Research Perspective. Suicide and Life-Threatening Behavior, 30, 8-17.

Jobes, D. A. (2006). Managing Suicidal Risk: A Collaborative Approach. New York: Guilford Press.

Jobes, D. A., Jacoby, A. M., Cimbolic, P., \& Hustead, L. A. T. (1997). Assessment and Treatment of Suicidal Clients in a University Counseling Center. Journal of Counseling Psychology, 44, 369-377. https://doi.org/10.1037/0022-0167.44.4.368

Jobes, D. A., Nelson, K. N., Peterson, E. M., Pentiuc, D., Downing, V., Francini, K., \& Kiernan, A. (2004). Describing Suicidality: An Investigation of Qualitative SSF Responses. Suicide and Life-Threatening Behavior, 34, 99-112.

https://doi.org/10.1521/suli.34.2.99.32788

Joiner, T., Pettit, J. W., Walker, R. L., Voelz, Z. R., Cruz, J., Rudd, M. D., \& Lester, D. (2002). Perceived Burdensomeness and Suicidality: Two Studies on the Suicide Notes of Those Attempting and Those Completing Suicide. Journal of Social \& Clinical Psychology, 21, 531-545. https://doi.org/10.1521/jscp.21.5.531.22624

Kovacs, M., \& Beck, A. T. (1977). The Wish to Die and the Wish to Live in Attempted Suicides. Journal of Clinical Psychology, 33, 361-365. https://doi.org/10.1002/1097-4679(197704)33:2<361::AID-JCLP2270330207>3.0.CO;2-H

Large, M., Myles, N., Myles, H., Corderoy, A., Wieser, M., Davidson, M., \& Ryan, C. J. (2018). Suicide Risk Assessment among Psychiatric Inpatients: A Systematic Review and Meta-Analysis of High-Risk Categories. Psychological Medicine, 48, 1119-1127. https://doi.org/10.1017/S0033291717002537

Linehan, M. M., Goodstein, J. L., Nielsen, S. L., \& Chiles, J. A. (1983). Reasons for Staying Alive When You Are Thinking of Killing Yourself: The Reasons for Living Inventory. Journal of Consulting and Clinical Psychology, 51, 276-286. https://doi.org/10.1037/0022-006X.51.2.276

Malone, K. M., Oquendo, M. A., Haas, G. L., Ellis, S. P., Li, S., \& Mann, J. J. (2000). Protective Factors against Suicidal Acts in Major Depression: Reasons for Living. American Journal of Psychiatry, 157, 1084-1088. https://doi.org/10.1176/appi.ajp.157.7.1084

Martin, D. J., Garsle, J. P., \& Davis, M. K. (2000). Relation of the Therapeutic Alliance with Outcome and Other Variables: A Meta-Analytic Review. Journal of Consulting and Clinical Psychology, 68, 43-450. https://doi.org/10.1037/0022-006X.68.3.438 
McCabe, R., \& Priebe, S. (2004). The Therapeutic Relationship in the Treatment of Severe Mental Illness: A Review of Methods and Findings. International Journal of Social Psychiatry, 50, 115-128. https://doi.org/10.1177/0020764004040959

Meerwijk, E. L., Parekh, A., Oquendo, M. A., Allen, I. E., Franck, L. S., \& Lee, K. A. (2016). Direct and Indirect Psychosocial and Behavioral Interventions to Prevent Suicide and Suicide Attempt: A Systematic Review and Metaanalysis. Lancet Psychiatry, 3, 544-554. https://doi.org/10.1016/S2215-0366(16)00064-X

Michel, K., \& Gysin-Maillart, A. (2015). ASSIP-Attempted Suicide Short Intervention Program: A Manual for Clinicians. Göttingen: Hogrefe Publishing. https://doi.org/10.1027/00476-000

Michel, K., \& Valach, L. (1997). Suicide as Goal-Directed Action. Archives of Suicide Research, 3, 213-221. https://doi.org/10.1080/13811119708258273

O’Connor, S. S., Jobes, D. A., Comtois, K. A., Atkins, D. C., Janis, K., Chessen, C. E., \& Landes, S. E. (2012b). Identifying Outpatients with Entrenched Suicidal Ideation Following Hospitalization. Suicide and Life-Threatening Behavior, 42, 173-184. https://doi.org/10.1111/j.1943-278X.2012.00080.x

O’Connor, S. S., Jobes, D. A., Yeargin, M. K., FitzGerald, M. E., Rodríguez, V. M., Conrad, A. K., \& Lineberry, T. W. (2012a). A Cross-Sectional Investigation of the Suicidal Spectrum: Typologies of Suicidality Based on Ambivalence about Living and Dying. Comprehensive Psychiatry, 53, 461-467. https://doi.org/10.1016/j.comppsych.2011.09.007

Pearson, J. L., Stanley, B., King, C. A., \& Fisher, C. B. (2001). Intervention Research with Persons at High Risk for Suicidality: Safety and Ethical Considerations. Journal of Clinical Psychiatry, 62, 17-26. https://doi.org/10.1037/e618682011-001

Priebe, S., Richardson, M., Cooney, M., Adedeji, O., \& McCabe, R. (2011). Does the Therapeutic Relationship Predict Outcomes of Psychiatric Treatment in Patients with Psychosis? A Systematic Review. Psychotherapy and Psychosomatics, 80, 70-77. https://doi.org/10.1159/000320976

Rickham, P. P. (1964). Human Experimentation. Code of Ethics of the World Medical Association. Declaration of Helsinki. BMJ, 2, 177.

https://doi.org/10.1136/bmj.2.5402.177

Ring, M., \& Gysin-Maillart, A. (2018). Under Review. Patients'Satisfaction with the Therapeutic Relationship and the Therapeutic Outcome Are Related to Suicide Ideation in the Attempted Suicide Short Intervention Program (ASSIP). Crisis.

Rudd, M. D., Bryan, C. J., Wertenberger, E. G., Peterson, A. L., Young-McCaughan, S., Mintz, J., Bruce, T. O. et al. (2015). Brief Cognitive-Behavioral Therapy Effects on Post-Treatment Suicide Attempts in a Military Sample: Results of a Randomized Clinical Trial with 2-Year Follow-Up. American Journal of Psychiatry, 172, 441-449. https://doi.org/10.1176/appi.ajp.2014.14070843

Rudd, M. D., Joiner, T., \& Rajab, M. H. (2001). Treating Suicidal Behavior: An Effective, Time-Limited Approach (Treatment Manuals for Practitioners). New York: Guilford.

Saltzman, C., Luetgert, M. J., Roth, C. H., Creaser, J., \& Howard, L. (1976). Formation of a Therapeutic Relationship: Experiences during the Initial Phase of Psychotherapy as Predictors of Treatment Duration and Outcome. Journal of Consulting and Clinical Psychology, 44, 546-555. https://doi.org/10.1037/0022-006X.44.4.546

Seligman, M. (1975). Helplessness: On Depression, Development and Death. San Francisco, CA: Freeman.

Shneidman, E. A. (1993). Suicide as Psychache: A Clinical Approach to Self-Destructive Behavior. Northvale, NJ: Aronson. 
Silverman, M. M., Berman, A. L., Sanddal, N. D., O’Carroll, P. W., \& Joiner, T. E. (2007). Rebuilding the Tower of Babel: A Revised Nomenclature for the Study of Suicide and Suicidal Behaviors, Part 1: Background, Rationale, and Methodology. Suicide and Life-Threatening Behavior, 37, 248-263. https://doi.org/10.1521/suli.2007.37.3.248

Wagner, B., \& Maercker, A. (2008). Deutsche Übersetzung der Beck Scale for Suicidal Ideation. Psychopathologie \& Klinische Intervention: Unveröffentlichtes Manuskript, Universität Zürich.

WHO (2014). ICD-10. World Health Organisation. http://www.who.int/classifications/icd/en

WHO (2017). Suicide Data. World Health Organisation. http://www.who.int/mental_health/prevention/suicide/suicideprevent/en

Yang, B., \& Lester, D. (2006). Recalculating the Economic Cost of Suicide. Death Studies, 31, 351-361. https://doi.org/10.1080/07481180601187209

Zhang, Y., Law, C. K., \& Yip, P. S. F. (2011). Psychological Factors Associated with the Incidence and Persistence of Suicidal Ideation. Journal of Affective Disorders, 133, 584-590. https://doi.org/10.1016/j.jad.2011.05.003

\section{Highlights}

- Good therapeutic alliance and high satisfaction with therapy success are associated with a stronger wish to live in participants in the Attempted Suicide Short Intervention Program-ASSIP.

- Individuals' internal struggle was related to lower rates of suicidal ideation over time.

- Individuals with lower internal struggle (more wish to die) require much more attention to prevent the risk of suicidal crises. 